\title{
Membrane Environment Enables Ultrafast Isomerization of Amphiphilic Azobenzene -INVITED
}

\author{
G. M. Paternò*1, E. Colombo2,3, V. Vurro1,4, F. Lodola1, V. Sesti5, F. Benfenati2,3, C. Bertarelli5, G. Lanzani1,4
}

\author{
1Center for Nano Science and Technology, Istituto Italiano di Tecnologia, Via Pascoli 70/3, 20133, Milano, Italy \\ 2 Center for Synaptic Neuroscience and Technology, Istituto Italiano di Tecnologia, Largo Rosanna Benzi 10, 16132 \\ Genova, Italy \\ 3IRCCS Ospedale Policlinico San Martino, Largo Rosanna Benzi 10, 16132 Genova, Italy \\ 4Dipartimento di Fisica, Politecnico di Milano, Piazza L. da Vinci 32, 20133 Milano, Italy \\ 5Dipartimento di Chimica, Materiali e Ingegneria Chimica "Giulio Natta", Politecnico di Milano, Piazza L. da Vinci 32, \\ 20133 Milano, Italy
}

\begin{abstract}
We report on the isomerization dynamics of an amphiphilic azobenzene that dwells within the plasma membrane. We found that in aggregates formed in water, the isomerization reaction is hindered, while radiative deactivation is favoured. However, once in the membrane, the molecule reacquires its isomerisation capacity. These data fully account for the recently reported experiments in neurons, showing that the amphiphilic azobenzenes are effective light actuators for the modification of the electrical state of the membrane.
\end{abstract}

\section{Introduction}

The precise, fast and efficient spatiotemporal control of cellular signalling via the use of external triggers has become an important topic in chemical biology ${ }^{1}$ and photopharmacology. ${ }^{2}$ In this regard, lightresponsive systems offer the possibility to employ light as a clean, non-invasive and spatio-temporally precise tool for controlling a variety of biological signals both in vitro and in vivo. ${ }^{3-5}$ Very recently a new amphiphilic azobenzene named ZIAPIN2, has been introduced. ZIAPIN2 predominantly localizes in the plasma membrane and potently modulates neuronal firing in vitro as well in vivo via an opto-mechanical effect. ${ }^{6}$ In order to fully characterize the photostimulation mechanism of ZIAPIN2 here we report a detailed investigation of its photophysics and dynamics in different media. ${ }^{7}$ By combining steady-state and time-resolved optical spectroscopies, we observed that ZIAPIN2 in water lends itself to a radiative transition likely mediated by the formation of excimers, whereas it reacquires its conformational dynamics in membrane mimicking environments. Characteristics time scales and reaction yields are reported, reconciling the fast response (ms) observed in functional studies and the apparent slow ensemble evolution (s) by determining the molecular conversion time in the tens of picosecond range. Finally, we validate this opto-mechanical phenomenon in a nonexcitable cell line that has an intrinsically low concentration of ion channels, to single out the effect on the plasma membrane itself.

\section{Results}

In order to fully characterize the photostimulation mechanism of ZIAPIN2 here we report a detailed investigation of its photophysics and dynamics in different media. We selected dimethyl sulfoxide (DMSO) that is a good solvent, as a control medium and vector for
ZIAPIN2, water that is the main component of extracellular and intracellular media and micelles of sodium dodecyl sulphate (SDS) to mimic the lipid bilayer environment. By combining steady-state and timeresolved optical spectroscopies, we observed that ZIAPIN2in water lends itself to a radiative transition likely mediated by the formation of excimers, whereas it reacquires its conformational dynamics in membrane mimicking environments (figure 1). Characteristics time scales and reaction yields are reported, reconciling the fast response (ms) observed in functional studies and the apparent slow ensemble evolution by determining the molecular conversion time in the tens of picosecond range. Finally, we validate this opto-mechanical phenomenon in a non-excitable cell line that has an intrinsically low concentration of ion channels, to single out the effect on the plasma membrane itself.

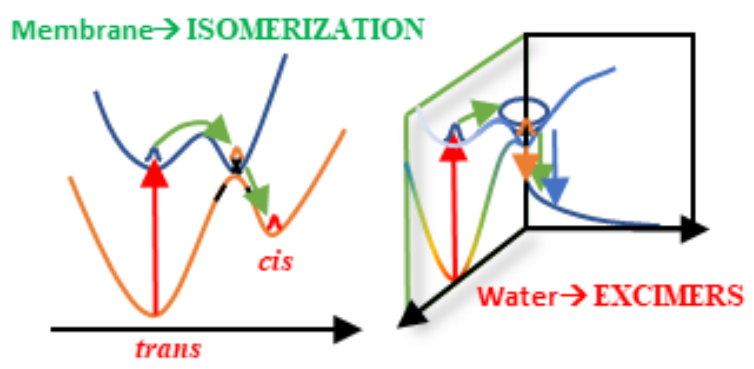

Fig. 1. Sketch of the photodynamics in DMSO, SDS and water as suggested by the TA data.

\section{Conclusions}

To summarise, the observed aggregation of photochromic molecules in water, where these azobenzenes lose their ability to photoisomerise, might represent an impediment to their

* Corresponding author: giuseppe.paterno@iit.it 
application in biology as water is the main component of biological environments. However, here we find that it is not true if the molecules have a preferential affinity for the cell membrane. In particular, we show that an amphiphilic azobenzene (ZIAPIN2) with non-covalent affinity for cell membranes can act as an efficient, fast and reversible modulator of the membrane potential via visible light. By applying steadystate and time-resolved ultrafast spectroscopies, we note that in water a strong aggregation occurs, effectively hampering photoisomerisation and opening up a deactivation path likely mediated by excimer formation. Alternatively, internalisation in the cell membrane prevents self-aggregation and restores the intrinsic ultrafast response of the photoswitch. This, ultimately allows light-triggering a large hyperpolarization in HEK293 cells whose magnitude follows the absorption profile of the trans state in membrane and, remarkably, permits to evoke biological photoinduced effects in vivo.[34] Such actuators are new tools that could contribute to the developing of opto-neuroscience, biophotonics and photopharmacology.

\section{References}

1. Beharry, A. A. \& Woolley, G. A. Chem. Soc. Rev. 40, 4422-4437 (2011).

2. Cabré, G. et al. Nat. Commun. 10, 907 (2019).

3. Parameswaran, R. et al. Nat. Nanotechnol. 13, 260-266 (2018).

4. Acarón Ledesma, H. et al. Nat. Nanotechnol. 14, 645-657 (2019).

5. Chen, S. Science (80-. ). 365, 456-457 (2019).

6. DiFrancesco, M. L. et al. Nat. Nanotechnol. (2020). doi:10.1038/s41565-019-0632-6

7. Paternò, G. M. et al. Adv. Sci. 7, 1903241 (2020). 